\title{
Feasibility study of the dynamic mesh model in FLUENT for fluid-structure interaction of a heart valve
}

\author{
K. Dumont ${ }^{1}$, J. Vierendeels ${ }^{2}$, P.R. Verdonck ${ }^{1}$ \\ ${ }^{1}$ IBITECH, Institute of Biomedical Technology, Hydraulics Laboratory, \\ Ghent University, Gent, Belgium \\ ${ }^{2}$ Department of Flow, Heat and Combustion Mechanics, Fluid Mechanics \\ Laboratory, Ghent University, Gent, Belgium
}

\begin{abstract}
A generic engineering example of fluid-structure interaction (FSI) is the motion of thin-walled, leaflet-like structure driven by fluid motion. A standard example in biomechanics is the opening and closing of aortic heart-valves, which is a delicate interaction between blood flow and geometrical and stiffness properties of the heart-valve leaflets. The coupled motion of flow and leaflet motion has been achieved in only a limited number of cases. In this paper a feasibility study of the dynamic mesh model in FLUENT is discussed for simulating heart valves during the ejection (systole). This study resulted in a simplified numerical model of the aortic valve hemodynamics. Although the results seem very promising, experimental validation is needed to prove the precision of the presented method.
\end{abstract}

\section{Introduction}

The interaction between blood and structure plays an important role in many cardiovascular problems. Examples are modeling of blood flow in the heart, vessels and in artificial organ design such as heart valves and cardiac assist devices [1]. Different methods have been used to model the blood flow in the cardiovascular system. Widespread is the arbitrary Lagrangian-Eulerian ALE method [2], commonly used for modeling blood flow in arteries [3]. The ALE method requires continuously updating the fluid mesh to accommodate for the motion of the structural domain. For modeling of heart valves this method is not very popular, because 
it is time-consuming. Makhijani et al are, according our knowledge, the only group presenting a real 3D simulation of a pericardial bioprosthetic aortic valve during the complete cardiac cycle [4]. The group of David et al [5] did some work on the dynamic analysis of bi-leaflet prosthetic mechanical heart valve. Also the fictitious domain formulation [6] has become more popular for the simulation of heart valves [7]. This method circumvents the fluid mesh updating according to the leaflet motion. The fluid is described using a fixed mesh in an Eulerian setting. The coupling of fluid and structure happens by so called Lagrange multipliers. Recently FLUENT Inc. [8] has released the dynamic mesh model for the simulation of flows where the shape of the domain is changing with time due to the motion of the domain boundaries. FLUENT allows to describe the motion of a boundary using user-defined functions (UDFs). This paper describes a numerical model of the aortic valve hemodynamics.

\section{Methods}

Figure 1 presents a flow diagram of the coupling of fluid and structure. In the following paragraphs the different steps of the scheme are explained.

\subsection{Geometry and boundary conditions of the aortic valve model}

A two dimensional geometry (figure 2) is used existing of an inflow tract (inlet $=$ left ventricle), a sinus of Valsalve (aortic valve) and an outflow tract (outlet $=$ aorta). On all the other walls no slip conditions are defined. As boundary conditions pulsatile aortic flow (in $\mathrm{m} / \mathrm{s}$ )

$$
v=\sin (\pi \cdot t)^{2}
$$

is given in function of the time $t$ at the inlet. At the outlet a constant gauge pressure of $0 \mathrm{~Pa}$ is assumed. For the valve leaflet the moment of inertia $I$ with the rotational axis along the edge of the leaflet can be calculated as $\operatorname{Eq}(2)$ :

$$
I=(1 / 3) \cdot m \cdot l^{2}
$$

with $m=\rho \cdot l \cdot t$ the mass of the leaflet per meter, $\rho$ the density of the leaflet $(1000$ $\left.\mathrm{kg} / \mathrm{m}^{3}\right), t$ the thickness of the leaflet $(1 \mathrm{~mm})$ and $l$ the length of the leaflet $(13 \mathrm{~mm})$. This results in $I=7.3 \cdot 10^{-7} \mathrm{~kg} \cdot \mathrm{m}^{2} / \mathrm{m}$.

\subsection{Finite volume package FLUENT Inc.}

For the integration of the Navier-Stokes equations in time a 1st order implicit scheme is used. Flow is supposed to be laminar and a constant dynamic viscosity of $4 \mathrm{mPa} \cdot \mathrm{s}$ for blood is used. Recently FLUENT Inc. has developed the DYNAMIC MESH model for the simulation of flows where the shape of the domain is changing with time due to the motion of the domain boundaries. FLUENT allows to describe the motion of a boundary using user-defined functions (UDFs). Using 


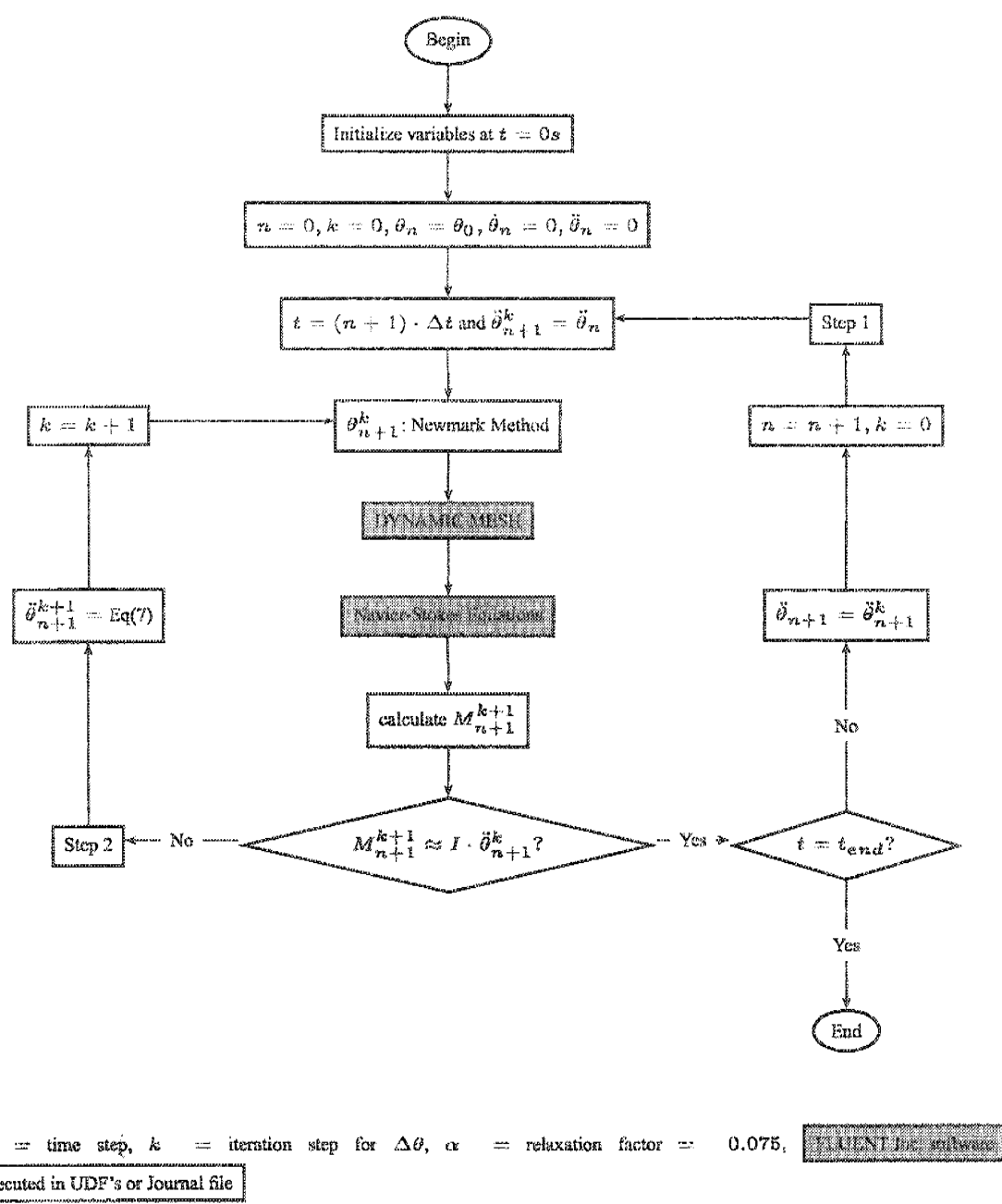

Figure 1: Flow diagram of the coupling of fluid and structure in FLUENT

these UDFs it is possible to calculate the movement of a valve leaflet using the equation $\mathrm{Eq}(3)$ :

$$
M=I \cdot \ddot{\theta}
$$

with $M$ the torque on a stiff valve leaflet, $I$ the moment of inertia and $\theta$ the opening angle of the leaflet as indicated in figure 2 . With the use of UDFs, a coupled solution of the leaflet motion/blood flow is obtained and it was possible to calculate the movement of the stiff leaflet during ejection (systole). The goal of this paper is to study the feasibility of the dynamic mesh model presented by FLUENT Inc. in FSI calculation (figure 1). 


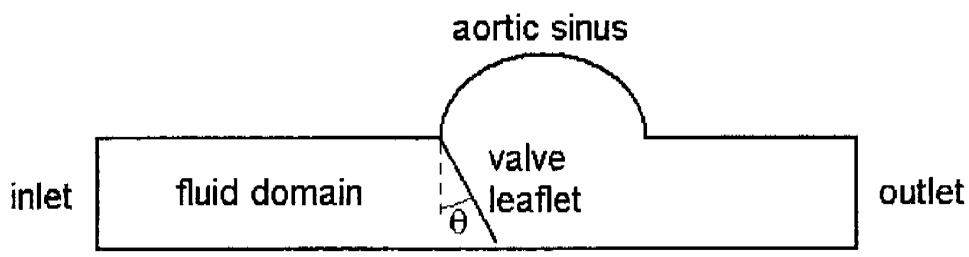

Figure 2: Geometry of 2D aortic valve model

\subsection{Newmark Method}

The subsequent position in function of time of the valve leaflet is calculated with the Newmark method (figure 1).

$$
\begin{aligned}
& \dot{\theta}_{n+1}=\dot{\theta}_{n}+(1-\gamma) \cdot \Delta t \cdot \ddot{\theta}_{n}+\gamma \Delta t \cdot \ddot{\theta}_{n+1} \\
& \theta_{n+1}=\theta_{n}+\Delta t \cdot \dot{\theta}_{n}+\left(\frac{1}{2}-\beta\right) \cdot \Delta t^{2} \cdot \ddot{\theta}_{n}+\beta \cdot \Delta t^{2} \cdot \ddot{\theta}_{n+1}
\end{aligned}
$$

We used $\gamma=\frac{1}{2}, \beta=\frac{1}{4}$. The indices $n+1$ and $n$ correspond with time levels $t+\Delta t$ and $t$ respectively.

\subsection{Iterative solution of the Newmark Method}

In order to obtain the position $\theta_{n+1}$ at $t+\Delta t$ an iterative approach is used. For each timestep there are $\mathrm{k}$ iterations performed in order to reach sufficient convergence of $\operatorname{Eq}(3)$.

\subsubsection{Step 1}

A first estimation ( $k=0$ ) of the new position $\theta_{n+1}$ is computed with the old value of the angular acceleration.

$$
\ddot{\theta}_{n+1}^{k=0}=\ddot{\theta}_{n}
$$

With this initial guess of the new position at $t+\Delta t$ the mesh is adapted with the DYNAMIC MESH model. After adaptation of the mesh, FLUENT is used to solve the Navier-Stokes equations. After convergence of the Navier-Stokes equations, $M_{n+1}^{k+1}$ is calculated and convergence of the $\mathrm{Eq}(6)$ is checked.

$$
\begin{gathered}
M_{n+1}^{k+1} \approx I \cdot \ddot{\theta}_{n+1}^{k} \\
\Downarrow \\
\left|M_{n+1}^{k+1}-I \cdot \ddot{\theta}_{n+1}^{k}\right|<\epsilon
\end{gathered}
$$




\subsubsection{Step 2}

When the correct position is not yet achieved, a better approximation of the angular acceleration is computed as follows:

$$
\ddot{\theta}_{n+1}^{k+1}=\ddot{\theta}_{n+1}^{k}+\omega \cdot\left(\frac{M_{n+1}^{k+1}}{I}-\ddot{\theta}_{n+1}^{k}\right)
$$

This formula uses $\omega$ as an underrelaxation factor, necessary to move in a stable way $(\omega \ll 1)$ to the correct position, rather than in large steps $(\omega=1)$. The value of $\omega$ used in this paper is 0.075 . With this better approximation of the angular velocity the new position is calculated, the mesh is adapted and the Navier-Stokes equations are solved. Again convergence of $\mathrm{Eq}(6)$ is checked.

Step 2 will be repeated until convergence of $\mathrm{Eq}(6)$ is achieved.

\section{Results and Discussion}

The presented method has been tested for $I=5 \cdot 10^{-7} \mathrm{~kg} \cdot \mathrm{m}^{2} / \mathrm{m}$. The value of $\Delta t=5 \cdot 10^{-5} \mathrm{~s}$ was determined by the dynamic mesh model of FLUENT. A large value of $\Delta t$ results in higher values of $\Delta \theta$ which resulted in negative volumes in the grid.

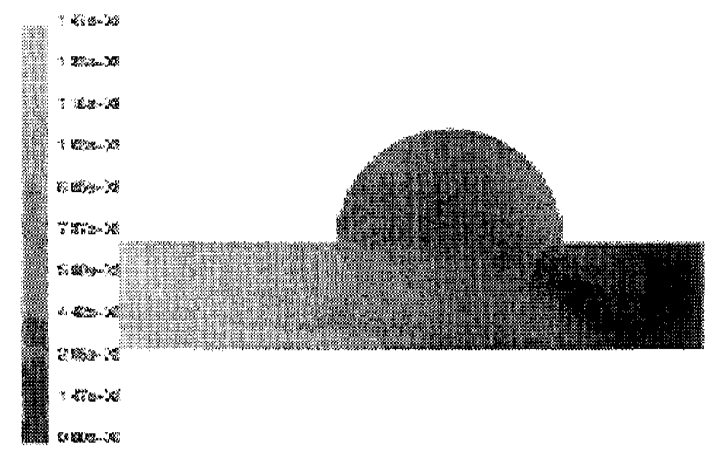

Figure 3: Pressure contours during the valve opening

Figure 3 shows pressure contours at the start of the ejection (systole), demonstrating the valvular pressure gradient $\Delta p=1470 \mathrm{~Pa} \simeq 11 \mathrm{mmHg}$. Figure 4 shows velocity contours for four different values of $t$ during the flow cycle. These figures show that the opening of the valve can be modelled using the presented method. It is clear that the valve is not completely closed at $t=0.1 \mathrm{~s}$. This is due to the fact that there is no regurgitation or leakage flow imposed at the inlet of the model. The regurgitation flow normally occurs in heart valves and plays an important role in the closure of a heart valve. At $t=0.075 \mathrm{~s}$ and $t=0.1 \mathrm{~s}$ a vortex is shown in the 

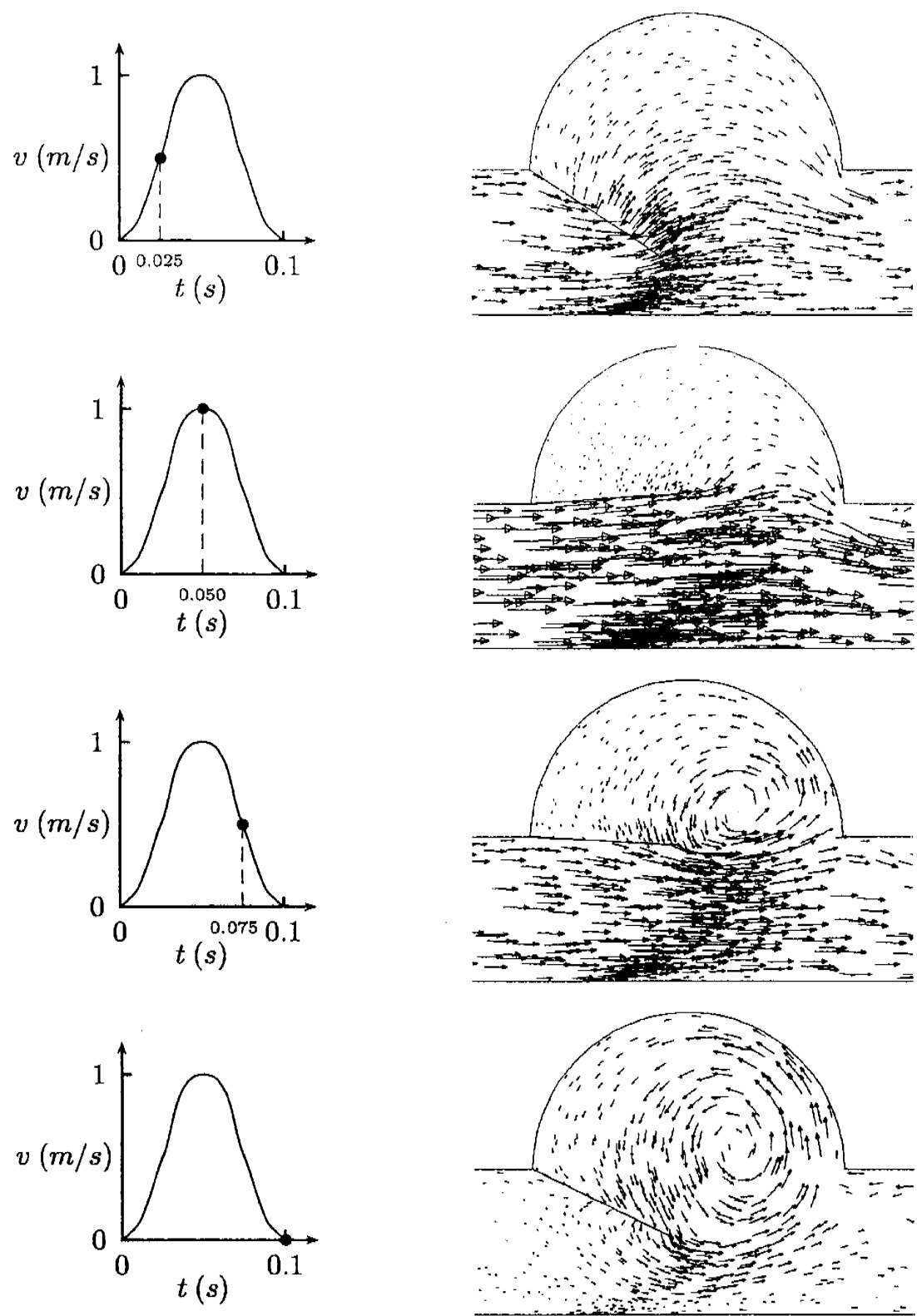

Figure 4: Velocity vectors for $t=0.025 \mathrm{~s}, t=0.050 \mathrm{~s}, t=0.075 \mathrm{~s}, t=0.100 \mathrm{~s}$

aortic sinus. At $t=0.054 \mathrm{~s}$ the leaflet has achieved a maximum angle of $95^{\circ}$. This is shown in figure 5 . 


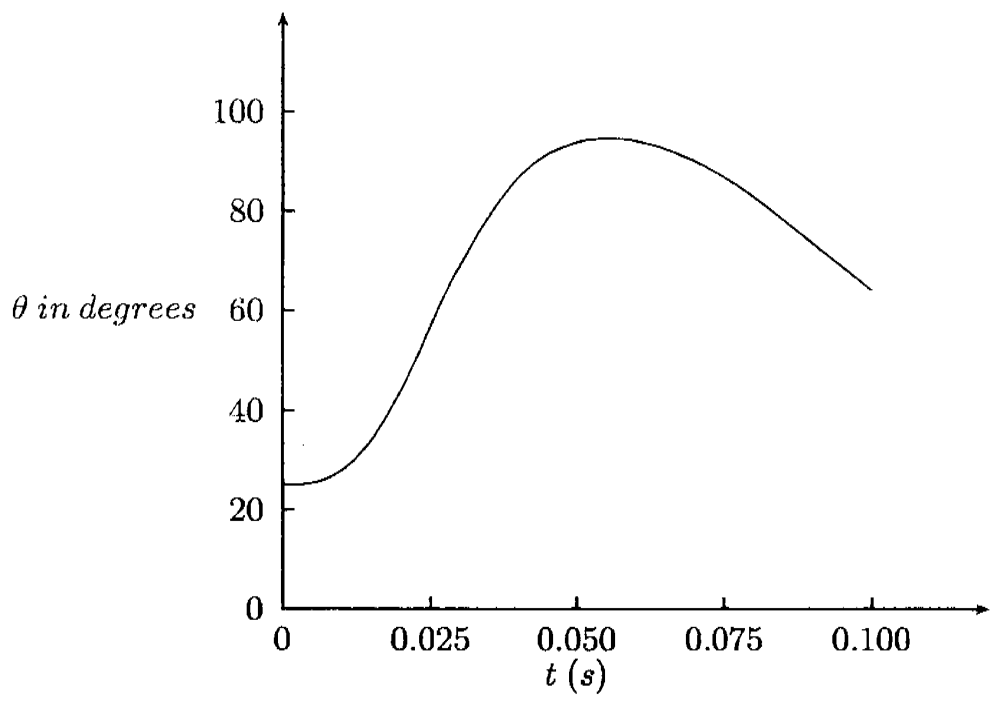

Figure 5: $\theta=$ angle of valve leaflet in function of time $t$

\section{Conclusions}

This study resulted in a simplified numerical model of the aortic valve hemodynamics. Although the results seem very promising, further development of the numerical model is necessary and experimental validation in a mock loop with Particle Image Velocimetry (PIV) is needed to prove the precision of the developed method. Future work will also include the assessment of flexible valve leaflets.

\section{Acknowledgements}

Kris Dumont is a recipient of a grant IWT-SB-3117 of the Flemish Institute for the Promotion of Scientific-Technological Research in Industry.

\section{References}

[1] Special issue: Computational fluid dynamics. Artificial Organs, 26(7), pp. 569-635, 2002.

[2] Hughes, T.J.R., Liu, W.K. \& Zimmerman, T.K., Lagragian-eulerian finite element formulation in incompressible viscous flows. Computer Methods in Applied Mechanics and Engineering, 29, pp. 329-349, 1991.

[3] Perktold, K., Hofer, M., Rappitsch, G., Loew, M., Kuban, B.D. \& Friedman, M.H., Validated computation of physiologic flow in a realistic coronary artery branch. Journal of Biomechanics, 31(3), pp. 217-228, 1998. 
[4] Makhijani, V.B., Yang, H.Q., Dionne, P.J. \& Thubrikar, M.J., Threedimensional coupled fluid-structure simulation of pericardial bioprosthetic aortic valve function. ASAIO Journal, 43(5), pp. M387-M392, 1997.

[5] David, T. \& Hsu, C.H., Dynamic analysis and geometry models for the design of bi-leaflet prosthetic mechanical heart valves. Medical Engineering \& Physics, 18(6), pp. 463-476, 1996.

[6] Baaijens, F.P.T., A fictitious domain/mortar element method for fluid-structure interaction. International Journal For Numerical Methods in Fluids, 35(7), pp. 743-761, 2001.

[7] De Hart, J., Peters, G.W.M., Schreurs, P.J.G. \& Baaijens, F.P.T., A twodimensional fluid-structure interaction model of the aortic value. Journal of Biomechanics, 33(9), pp. 1079-1088, 2000.

[8] Fluent inc., http://www.fluent.com/, 2002. 